benefit in metastatic colorectal cancer: a meta-analysis of randomized, controlled trials. Ann Oncol 26: 13-21.

Stintzing S, Jung A, Rossius L, Modest DP, von Weikersthal LF, Decker T, Kiani A, Al-Batran SE, Vehling-Kaiser U, Heintges T, Moehler M, Scheithauer W, Kirchner T, Heinemann V (2014) Mutations within the
EGFR signaling pathway: Influence on efficacy in FIRE-3-A randomized phase III study of FOLFIRI plus cetuximab or bevacizumab as first-line treatment for wild-type (WT) KRAS (exon 2) metastatic colorectal cancer (mCRC) patients. J Clin Oncol 32(suppl 3): abstr 445 .

*Correspondence: F Pietrantonio; E-mail: filippo.pietrantonio@istitutotumori.mi.it

Published online 10 September 2015

(c) 2015 Cancer Research UK. All rights reserved 0007 - 0920/15
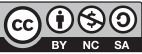

http://creativecommons.org/licenses/by-nc-sa/4.0/

\title{
Reply: Comment on 'Meta-analysis of BRAF mutation as a predictive biomarker of benefit from anti-EGFR monoclonal-antibody therapy for RAS wild-type metastatic colorectal cancer'
}

\author{
Andrew Rowland ${ }^{\star}, 1,2$, Mafalda M Dias ${ }^{1,3}$, Michael D Wiese ${ }^{3}$, Ganessan Kichenadasse ${ }^{2}$, Ross A McKinnon², Christos S Karapetis ${ }^{2}$ \\ and Michael J Sorich ${ }^{1,2}$
}

\begin{abstract}
${ }^{1}$ Department of Clinical Pharmacology, School of Medicine, Flinders University, Adelaide 5042, South Australia, Australia; ${ }^{2}$ Flinders Centre for Innovation in Cancer, School of Medicine, Flinders University, Adelaide 5042, South Australia, Australia and ${ }^{3}$ School of Pharmacy and Medical Sciences, University of South Australia, Adelaide 5000, South Australia, Australia
\end{abstract}

Sir,

We thank Pietrantonio et al, for their interest in our recent manuscript (Rowland et al, 2015). We also acknowledge their mutual interest in the field, and share their goal of informing clinicians with the most accurate and complete evidence to ensure that patients with metastatic colorectal cancer (mCRC) are both afforded access and guided to the most appropriate treatment interventions.

Pietrantonio et al, highlight that the power to detect differences in treatment effect between subgroups based on BRAF mutation status is often poor, and imply that the risk of false negative results is not properly acknowledged in this manuscript. As described in the discussion section of the manuscript, we have clearly and prominently addressed the issue of false positive and negative results (Rowland et al, 2015). We believe that the evaluation of the predictive nature of BRAF mutation for anti-EGFR therapy highlights what may be a relatively common scenario in which it will be difficult to make definitive conclusions despite having results from a number of high quality secondary analyses of clinical trials. Factors contributing to the substantial risk of false positive or false negative conclusions in this setting include (i) the post hoc nature of the analyses and the associated difficulty in correcting for multiple hypotheses testing, (ii) the biomarker having a low prevalence impacting on the precision of the estimates, (iii) there being significant statistical heterogeneity (inconsistency) in results between clinical studies, and (iv) that this biomarker may have a more modest impact (e.g. attenuate than annul) on treatment efficacy compared to the prominent biomarkers that have made their way into routine clinical practice such as RAS mutations (Sorich et al, 2014).

Pietrantonio et al. highlight their own meta-analysis of anti-EGFR mAb therapy in BRAF mutant tumours (Pietrantonio et al, 2015). This meta-analysis concludes that ' $\mathrm{C}$ - or P-based therapy did not increase the benefit of standard therapy or the BSC in RAS-wt/BRAF-mut CRC patients.' This meta-analysis principally differs from ours on the basis of the methodology used to evaluate whether BRAF is a predictive marker of anti-EGFR mAb efficacy. The evaluation of heterogeneity of effect between subgroups by a test of interaction is the standard approach recommended, on the basis that evaluating the efficacy of a treatment with respect to an isolated subgroup is well known to have a high risk of false positive results (i.e. falsely concluding that a subgroup has no effect) (Rothwell, 2005; Kent et al, 2010; Sun et al, 2010). The Pietrantonio meta-analysis only evaluated anti-EGFR efficacy in the BRAF mutant subgroup, whereas our study compared the efficacy in the BRAF mutant subgroup to the subgroup without a BRAF mutation (see (Altman and Matthews, 1996; Matthews and Altman, 1996) for a simple introduction to the concept of interaction). With respect to significance level, in our experience a stricter rather than more lenient significance level is often preferred for making strong claims that will have significant clinical and policy implications (as compared with exploratory/ screening questions). This is due to the post hoc nature of many subgroup analyses and the inflated risks of false positives with multiple hypotheses testing which are generally not explicitly adjusted for (Rothwell et al, 2005).

Our analysis highlight that the evidence for there being a treatment effect difference between BRAF subgroups does not meet the conventional levels of evidence when evaluated using the generally accepted approach for evaluating subgroup differences in RCTs-hence our more moderate conclusion that there currently is insufficient evidence to definitively state that there is a reduced (or no) benefit for individuals with mutated BRAF. Cognizant of the risk of false negative results, we have not ruled out the possibility that BRAF mutation status influences anti-EGFR therapy efficacy, merely that the evidence does not support a definitive claim that BRAF mutations does impact on efficacy. We advocate that as we can neither definitively claim or rule out a predictive effect of a BRAF mutation that it should remain at the clinician's and patient's discretion to decide whether to test for BRAF mutation and whether use of an anti-EGFR $\mathrm{mAb}$ is appropriate for a specific patient with a BRAF mutant tumor. We are concerned that the conclusion of the Pietrantonio meta-analysis of no benefit for the BRAF mutant subgroup may inadvertently lead to reduced clinician discretion to treat patients with BRAF mutant tumours. For example, if the evidence clearly indicated that anti-EGFR mAbs do not have benefit for patients with BRAF mutant tumours, then in many jurisdictions this would lead payers to restrict subsidy of anti-EGFR mAbs to individuals without a BRAF mutation (with routine testing for BRAF mutations). We do not believe that the evidence currently available supports with sufficient certainty that such individuals do not receive any benefit from anti-EGFR mAb therapy.

We agree with Pietrantonio et al, that the trials comparing bevacizumab to anti-EGFR $\mathrm{mAb}$ therapy are very informative in terms of guiding contemporary first line therapy in mCRC and that when results stratified by BRAF mutations status become available they will provide additional useful insight into the impact of BRAF mutations on anti-EGFR mAb therapy. However, it will be important to carefully manage how this data is analysed in conjunction with the data from trials that evaluate the addition of anti-EGFR therapy to standard therapy (e.g. focus on the difference between subgroups (Sorich et al, 2014)) as pooling results from these different types of trials in a meta-analysis focusing only on the effect size in the BRAF mutant subgroup may be misleading (Rowland et al, 2015).

On behalf of my colleagues

Kind Regards

Dr A Rowland

\section{CONFLICT OF INTEREST}

The authors declare no conflict of interest.

\section{REFERENCES}

Altman DG, Matthews JNS (1996) Statistics Notes: interaction 1: heterogeneity of effects. BMJ 313: 808.

Kent DM, Rothwell PM, Ioannidis JP, Altman DG, Hayward RA (2010) Assessing and reporting heterogeneity in treatment effects in clinical trials: a proposal. Trials 11: 85 .

Matthews JNS, Altman DG (1996) Statistics Notes: Interaction 2: compare effect sizes not $\mathrm{P}$ values. $B M J$ J13: 313 .

Pietrantonio F, Petrelli F, Coinu A, Di Bartolomeo M, Borgonovo K, Maggi C Cabiddu M, Iacovelli R, Bossi I, Lonati V, Ghilardi M, de Braud F, Barni S (2015) Predictive role of BRAF mutations in patients with advanced colorectal cancer receiving cetuximab and panitumumab: a meta-analysis. Eur J Cancer 51: $587-594$.

Rothwell PM (2005) Treating individuals 2. Subgroup analysis in randomised controlled trials: importance, indications, and interpretation. Lancet 365: $176-186$.

Rowland A, Dias MM, Wiese MD, Kichenadasse G, McKinnon RA, Karapetis CS, Sorich MJ (2015) Meta-analysis of BRAF mutation as a predictive biomarker of benefit from anti-EGFR monoclonal antibody therapy for RAS wild-type metastatic colorectal cancer. Br J Cancer 112: 1888-1894.

Sorich MJ, Wiese MD, Rowland A, Kichenadasse G, McKinnon RA, Karapetis CS (2014) Extended RAS mutations and anti-EGFR monoclonal antibody survival benefit in metastatic colorectal cancer: a meta-analysis of randomized controlled trials. Ann Oncol 26: 13-21.

Sun X, Briel M, Walter SD, Guyatt GH (2010) Is a subgroup effect believable? Updating criteria to evaluate the credibility of subgroup analyses. $B M J$ 340: c117

${ }^{\star}$ Correspondence: Dr A Rowland; E-mail: andrew.rowland@flinders.edu.au Published online 10 September 2015

(c) 2015 Cancer Research UK. All rights reserved 0007-0920/15

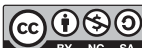

